Open J. Math. Sci., Vol. 1(2017), No. 1, pp. 72 - 84

Website: https://pisrt.org/psr-press/journals/oms/

ISSN: 2523-0212 (Online) 2616-4906 (Print)

http://dx.doi.org/10.30538/oms2017.0008

\title{
KAUFFMAN BRACKET OF 2- AND 3-STRAND BRAID LINKS
}

\author{
ABDUL RAUF NIZAMI ${ }^{1}$
}

\begin{abstract}
In this paper we give explicit formulas of the Kauffman bracket of the 2-strand braid link $\widehat{x_{1}^{n}}$ and the 3 -strand braid link $\widehat{x_{1}^{b} x_{2}^{m}}$. We also show that the Kauffman bracket of the 3 -strand braid link $\widehat{x_{1}^{b} x_{2}^{m}}$ is actually the product of the Kauffman brackets of the 2-strand braid links $\widehat{x_{1}^{b}}$ and $\widehat{x_{1}^{m}}$.
\end{abstract}

AMS Mathematics Subject Classification: 57M27, 57Q45.

Key words and phrases: kauffman bracket; braid link.

\section{Introduction}

The Kauffman bracket was introduced by L. H. Kauffman in 1987 in [1].

The Kauffman bracket (polynomial) is actually not a knot invariant because it is not invariant under the first Reidemeister move. However, it has many applications and it can be extended to the popular Jones polynomial, which is invariant under all three Reidemeister moves. In the present work we shall confine ourselves to the Kauffman bracket to avoid from unnecessary length and to leave it for applications. In [2] Nizami et al, computed Khavanov Homology of Braid Links and in [3] gave recursive form of Kauffman Bracket.

This paper is organized as follows: In Section 2 we shall give the basic ideas about knots, braids, and the Kauffman bracket. In Section 3 we shall present the main results.

Received 1 September 2017 . Revised 1 October 2017.

1 Corresponding Author

${ }^{\dagger}$ This work is supported by the Higher Education Commission, Pakistan

(C) 2017 Abdul Rauf Nizami. This is an open access article distributed under the Creative Commons Attribution License, which permits unrestricted use, distribution, and reproduction in any medium, provided the original work is properly cited. 


\section{Preliminary Notions}

2.1. Links. A link is a disjoint union of circles embedded in $\mathbb{R}^{3}$. A onecomponent link is called a knot. Links are usually studied via projecting them on a plan; a projection with extra information of overcrossing and undercrossing is called the link diagram.

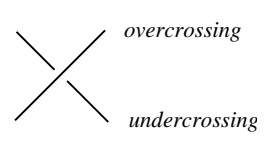

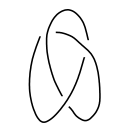

Trefoil knot

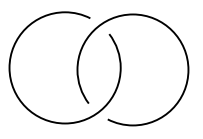

Hopf link

Two links are isotopic iff one of them can be transformed to the other by a diffeomorphism of the ambient space onto itself. A fundamental result by Reidemeister [4] about the isotopic link diagrams is: Two unoriented links $L_{1}$ and $L_{2}$ are equivalent if and only if a diagram of $L_{1}$ can be transformed into a diagram of $L_{2}$ by a finite sequence of ambient isotopies of the plane and the local (Reidemeister) moves of the following three types:

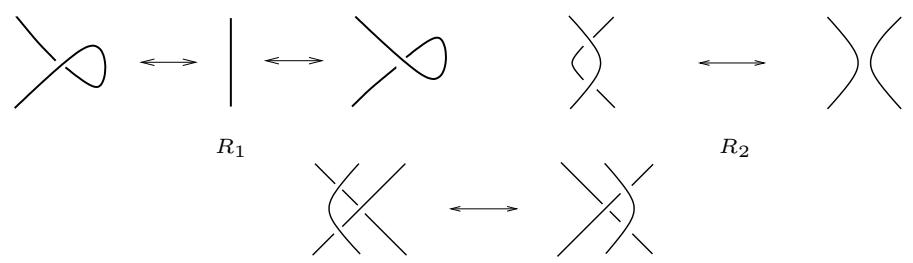

$R_{3}$

The set of all links that are equivalent to a link $L$ is called a class of $L$. By a link $L$ we shall always mean the class of $L$.

The main question of knot theory is which two links are equivalent and which are not? To address this question one needs a knot invariant, a function that gives one value on all links that belong to a single class and gives different values (but not always) on knots that belong to different classes. The present work is basically concerned with this question.

2.2. Braids. Braids were first studied by Emil Artin in $1925[5,6]$, which play an important role in knot theory, see $[7,8]$ for detail.

An $n$-strand braid is a set of $n$ non intersecting smooth paths connecting $n$ points on a horizontal plane to $n$ points exactly below them on another horizontal plane in an arbitrary order. The smooth paths are called strands of the braid.

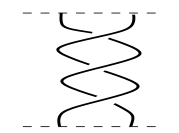

A 2-strand braid

The product $a b$ of two $n$-strand braids is defined by putting $b$ above $a$ and gluing their end points.

A braid with only one crossing is called elementary braid. The $i$ th elementary braid $x_{i}$ on $n$ strands is: 


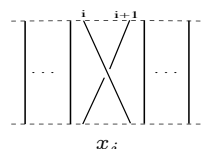

A useful property of elementary braids is that every braid can be written as a product of elementary braids. For instance, the above 2 -strand braid is $x_{i}^{-3}=$ $\left(x_{i}^{-1}\right)\left(x_{i}^{-1}\right)\left(x_{i}^{-1}\right)$.

The closure of a braid $b$ is the link $\widehat{b}$ obtained by connecting the lower ends of $b$ with the corresponding upper ends.

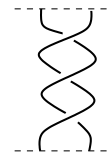

$b$

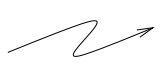

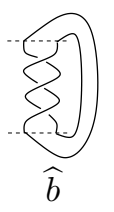

An important result by Alexander [9] connecting knots and braids is: Each link can be represented as the closure of a braid.

Remark 2.1. In the last section we shall present all the links as closures of products of elementary braids.

2.3. The Kauffman Bracket. The Kauffman bracket was introduced by Kauffman in [10].

Before the definition it is better to understand the two types of splitting of a crossing, the $A$-type and the $B$-type splittings:

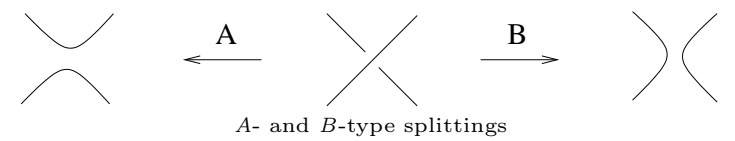

In the following, the symbols $\bigcirc$ and $\bigsqcup$ represent respectively the unknot and the disconnected sum.

Definition 2.1. The Kauffman bracket is the function $\langle\cdot\rangle:$ Links $\rightarrow \mathbb{Z}\left[a, a^{-1}\right]$ defined by the axioms:

$$
\begin{aligned}
\langle L\rangle & =a\left\langle L_{A}\right\rangle+a^{-1}\left\langle L_{B}\right\rangle \\
\langle L \sqcup \bigcirc\rangle & =\left(-a^{2}-a^{-2}\right)\langle L\rangle \\
\langle\bigcirc\rangle & =1
\end{aligned}
$$

Here $L, L_{A}$, and $L_{B}$ are three links which are isotopic everywhere except at one crossing where the look as in the figure:
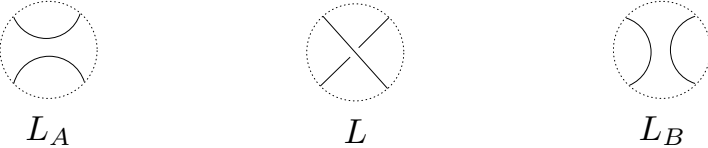

Proposition 2.2. The Kauffman polynomial is invariant under second and third Reidemeister moves but not under the first Reidemeister move. 


\section{Main Results}

In this section we shall give the Kauffman bracket of the links $\widehat{x_{1}^{n}}$ and $\widehat{x_{1}^{b} x_{2}^{m}}$, and show that $\left\langle\widehat{x_{1}^{b} x_{2}^{m}}\right\rangle=\left\langle\widehat{x_{1}^{b}}\right\rangle\left\langle\widehat{x_{2}^{m}}\right\rangle$.

The links $\widehat{x_{1}^{n}}$ fall into two categories, the two-component links when $n$ is even and the one-component links (means knots) when $n$ is odd. When $n$ is even, we have:

Proposition 3.1. The Kauffman bracket of the link $\widehat{x_{1}^{n}}$, when $n \geq 2$ is even, is

$$
<\widehat{x_{1}^{n}}>=-a^{3 n-2}+a^{3 n-6}-a^{3 n-10}+a^{3 n-14}-\cdots-a^{-n+6}-a^{-n-2} .
$$

Proof. We prove it by induction on $n$.

When $n=2$,

$$
\begin{aligned}
\left\langle\widehat{x_{1}^{2}}\right\rangle & =\langle\Omega\rangle=a\langle\Omega\rangle+a^{-1}\langle\Omega\rangle \\
& =a\left[a\langle\supseteq]_{\rangle}+a^{-1}\langle\Omega\rangle\right]+a^{-1}\left[a\langle\Omega\rangle+a^{-1}\langle\Omega\rangle\right] \\
& =a\left[a\left(-a^{2}-a^{-2}\right)+a^{-1}(1)\right]+a^{-1}\left[a(1)+a^{-1}\left(-a^{2}-a^{-2}\right)\right] \\
& =-a^{4}-a^{-4} .
\end{aligned}
$$

Similarly, we have

$$
\begin{aligned}
\widehat{\left\langle x_{1}^{4}\right\rangle} & =-a^{10}+a^{6}-a^{2}-a^{-6} \\
& \left.=-a^{3(4)-2}+a^{3(4)-6}+a^{-2} \widehat{\left\langle x_{1}^{2}\right.}\right\rangle
\end{aligned}
$$

and

$$
\begin{aligned}
\widehat{\left.x_{1}^{6}\right\rangle} & =-a^{16}+a^{12}-a^{8}+a^{4}-a^{0}-a^{-8} \\
& =-a^{3(6)-2}+a^{3(6)-6}+a^{-2}\left\langle\widehat{x_{1}^{4}}\right\rangle .
\end{aligned}
$$

We now assume the result holds for $n=k$, that is

$$
\left\langle\widehat{x_{1}^{k}}\right\rangle=-a^{3 k-2}+a^{3 k-6}-a^{3 k-10}+a^{3 k-14}-\cdots-a^{6-k}-a^{-k-2} .
$$

Now for $n=k+1$, we, following Equations 3.3 and 3.4, write

$$
\begin{aligned}
\widehat{\left.x_{1}^{k+2}\right\rangle}= & -a^{3(k+2)-2}+a^{3(k+2)-6}+a^{-2}\left\langle\widehat{x_{1}^{k}}\right\rangle \\
= & -a^{3(k+2)-2}+a^{3(k+2)-6}+a^{-2}\left[-a^{3 k-2}+a^{3 k-6}-a^{3 k-10}\right. \\
& \left.+a^{3 k-14}-\cdots-a^{6-k}-a^{-k-2}\right] \\
= & -a^{3(k+2)-2}+a^{3(k+2)-6}-a^{3 k-4}+a^{3 k-8}-a^{3 k-12}+a^{3 k-16} \\
& -\cdots-a^{4-k}-a^{-k-4} \\
= & -a^{3(k+2)-2}+a^{3(k+2)-6}-a^{3(k+2)-10}+a^{3(k+2)-14}-a^{3(k+2)-18} \\
& +a^{3(k+2)-22}-\cdots-a^{6-(k+2)}-a^{-(k+2)-2}
\end{aligned}
$$


This completes the proof.

Proposition 3.2. The Kauffman bracket of the knots $\widehat{x_{1}^{n}}$, when $n$ is odd, is

$$
<\widehat{x_{1}^{n}}>=a^{3 n-2}-a^{3 n-6}+a^{3 n-10}-a^{3 n-14}+\cdots-a^{-n+6}-a^{-n-2} .
$$

Proof. Similar to the proof of Proposition 3.1

Proposition 3.3. The Kauffman bracket of the braid link $\widehat{x_{1}^{b} x_{2}^{b}}$, when $b$ is even, is

$$
\begin{aligned}
\left\langle\widehat{x_{1}^{b} x_{2}^{b}}\right\rangle= & \sum_{i=1}^{b-1} i(-1)^{i+1} a^{6 b-4 i}+\sum_{i=1}^{b}(-1)^{i+1}(b-i) a^{2 b-4 i}-(b-2) a^{2 b} \\
& +a^{4-2 b}+a^{-2 b-4}
\end{aligned}
$$

Proof. We prove it by induction on $b$.

When $b=2$, we have

$$
\begin{aligned}
& \left.\widehat{\left\langle x_{1}^{2} x_{2}^{2}\right.}\right\rangle=\langle\sqrt{\Omega}\rangle=a\langle\sqrt{\Omega}\rangle+a^{-1}\langle[\Omega
\end{aligned}
$$

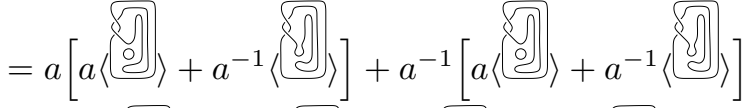

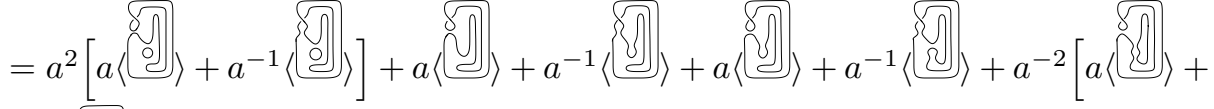

$$
\begin{aligned}
& a^{-1}\langle\{j\}]
\end{aligned}
$$

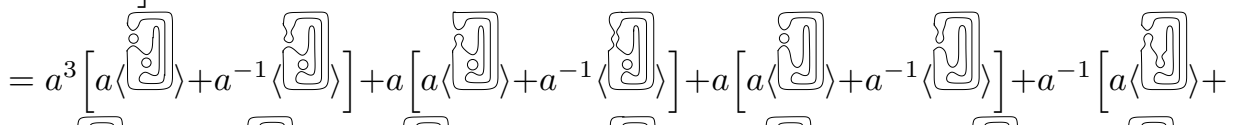

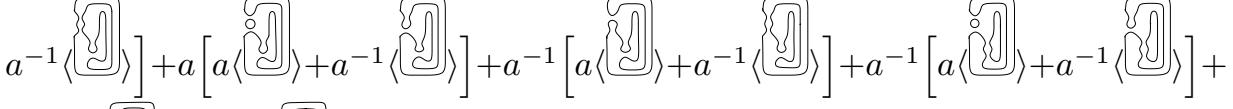

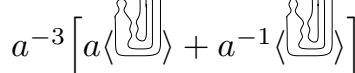

$$
\begin{aligned}
& =a^{4}\left(-a^{2}-a^{-2}\right)^{2}+a^{2}\left(-a^{2}-a^{-2}\right)+a^{2}\left(-a^{2}-a^{-2}\right)+\left(-a^{2}-a^{-2}\right)^{2}+a^{2}\left(-a^{2}-\right. \\
& \left.a^{-2}\right)+1+1+a^{-2}\left(-a^{2}-a^{-2}\right)+a^{2}\left(-a^{2}-a^{-2}\right)+1+1+a^{-2}\left(-a^{2}-a^{-2}\right)+ \\
& \left(-a^{2}-a^{-2}\right)^{2}+a^{-2}\left(-a^{2}-a^{-2}\right)+a^{-2}\left(-a^{2}-a^{-2}\right)+a^{-4}\left(-a^{2}-a^{-2}\right)^{2} \\
& =a^{8}+2+a^{-8}=\left[a^{8}\right]+[1]+[0]+\left[1+a^{-8}\right] \\
& =\sum_{i=1}^{1} i(-1)^{i+1} a^{6(2)-4 i}+\sum_{i=1}^{2}(-1)^{i+1}(2-i) a^{2(2)-4 i}-(2-2) a^{2(2)}+a^{4-2(2)}+ \\
& \text { as required. }
\end{aligned}
$$

Similarly, we get

$$
\begin{aligned}
\left.\widehat{x_{1}^{4} x_{2}^{4}}\right\rangle & =a^{20}-2 a^{16}+3 a^{12}-2 a^{8}+3 a^{4}-2+2 a^{-4}+a^{-12} \\
& =\left[a^{20}-2 a^{16}+3 a^{12}\right]+\left[3 a^{4}-2+a^{-4}\right]-2 a^{8}+\left[a^{-4}+a^{-12}\right]
\end{aligned}
$$




$$
\begin{aligned}
= & \sum_{i=1}^{3} i(-1)^{i+1} a^{24-4 i}+\sum_{i=1}^{4}(-1)^{i+1}(4-i) a^{8-4 i}-2 a^{8} \\
& +a^{-4}+a^{-12} .
\end{aligned}
$$

In order to manage the proof, we reorganize (3.7):

$$
\begin{aligned}
\widehat{\left\langle x_{1}^{4} x_{2}^{4}\right\rangle}= & {\left[a^{4}+2 a^{-4}+a^{-12}\right]-\left[a^{4}\right]+\left[a^{20}\right]-2+\left[-2 a^{16}+3 a^{12}\right] } \\
& +\left[-2 a^{8}+3 a^{4}\right] \\
= & a^{-4}\left[\widehat{\left.x_{1}^{2} x_{2}^{2}\right\rangle}\right]-\sum_{i=1}^{1} i(-1)^{i+1} a^{8-4 i}+\sum_{i=1}^{1} i(-1)^{i+1} a^{24-4 i}-2 \\
& +\sum_{i=2}^{3} i(-1)^{i+1} a^{24-4 i}-2 a^{8}+3 a^{4}
\end{aligned}
$$

Similarly,

$$
\begin{aligned}
\widehat{\left\langle x_{1}^{6} x_{2}^{6}\right\rangle}= & a^{32}-2 a^{28}+3 a^{24}-4 a^{20}+5 a^{16}-4 a^{12}+5 a^{8}-4 a^{4}+3-2 a^{-4} \\
& +2 a^{-8}+a^{-16} \\
= & a^{-4}\left[\widehat{\left\langle x_{1}^{4} x_{2}^{4}\right\rangle}\right]-\sum_{i=1}^{3} i(-1)^{i+1} a^{20-4 i}+\sum_{i=1}^{3} i(-1)^{i+1} a^{36-4 i}-2 a^{4} \\
& +\sum_{i=4}^{5} i(-1)^{i+1} a^{36-4 i}-4 a^{12}+5 a^{8}
\end{aligned}
$$

Deducting from Equations 3.9 and 3.10 , we can write

$$
\begin{aligned}
& \left.\widehat{\left\langle x_{1}^{b} x_{2}^{b}\right.}\right\rangle=a^{-4}\left[\left\langle x_{1}^{b-2} x_{2}^{b-2}\right\rangle\right]-\sum_{i=1}^{b-3} i(-1)^{i+1} a^{6 b-4 i-16}+\sum_{i=1}^{b-3} i(-1)^{i+1} a^{6 b-4 i} \\
& -2 a^{2 b-8}+\sum_{i=b-2}^{b-1} i(-1)^{i+1} a^{6 b-4 i}-(b-2) a^{2 b}+(b-1) a^{2 b-4} .
\end{aligned}
$$

We now assume the result holds for $b=k$, that is

$$
\begin{aligned}
\left.\widehat{x_{1}^{k} x_{2}^{k}}\right\rangle= & \sum_{i=1}^{k-1} i(-1)^{i+1} a^{6 k-4 i}+\sum_{i=1}^{k}(-1)^{i+1}(k-i) a^{2 k-4 i}-(k-2) a^{2 k} \\
& +a^{4-2 k}+a^{-2 k-4} .
\end{aligned}
$$

Now for $b=k+2$, we have

$$
\begin{aligned}
\left\langle x_{1}^{k+2} x_{2}^{k+2}\right\rangle= & a^{-4}\left[\left\langle\widehat{x_{1}^{k} x_{2}^{k}}\right\rangle\right]-\sum_{i=1}^{k-1} i(-1)^{i+1} a^{6 k-4 i-4}+\sum_{i=1}^{k-1} i(-1)^{i+1} a^{6 k-4 i+12} \\
& -2 a^{2 k-4}+\sum_{i=k}^{k+1} i(-1)^{i+1} a^{6 k-4 i+12}-k a^{2 k+4}+(k+1) a^{2 k}
\end{aligned}
$$




$$
\begin{aligned}
= & \sum_{i=1}^{k-1} i(-1)^{i+1} a^{6 k-4 i-4}+\sum_{i=1}^{k}(-1)^{i+1}(k-i) a^{2 k-4 i-4} \\
& -(k-2) a^{2 k-4}+a^{-2 k}+a^{-2 k-8} \\
& -\sum_{i=1}^{k-1} i(-1)^{i+1} a^{6 k-4 i-4}+\sum_{i=1}^{k-1} i(-1)^{i+1} a^{6 k-4 i+12}-2 a^{2 k-4} \\
& +\sum_{i=k}^{k+1} i(-1)^{i+1} a^{6 k-4 i+12}-k a^{2 k+4}+(k+1) a^{2 k} \\
= & \sum_{i=1}^{k+1} i(-1)^{i+1} a^{6 k-4 i+12}+\sum_{i=-1}^{k}(-1)^{i+1}(k-i) a^{2 k-4 i-4} \\
& +a^{-2 k}+a^{-2 k-8}-k a^{2 k+4} \\
= & \sum_{i=1}^{k+1} i(-1)^{i+1} a^{6 k-4 i+12}+\sum_{i=1}^{k+2}(-1)^{i+1}(k+2-i) a^{2 k-4 i+4} \\
& +a^{-2 k}+a^{-2 k-8}-k a^{2 k+4}
\end{aligned}
$$

and the induction is completed.

Proposition 3.4. The Kauffman bracket of the braid link $\widehat{x_{1}^{b} x_{2}^{b}}$, when $b$ is odd, is

$$
\begin{aligned}
\left\langle\widehat{x_{1}^{b} x_{2}^{b}}\right\rangle= & \sum_{i=1}^{b-1} i(-1)^{i+1} a^{6 b-4 i}+\sum_{i=1}^{b}(-1)^{i}(b-i) a^{2 b-4 i}+(b-2) a^{2 b} \\
& +a^{4-2 b}+a^{-2 b-4} .
\end{aligned}
$$

Proof. Similar to the proof of proposition 3.3.

Proposition 3.5. The Kauffman bracket of $\widehat{x_{1}^{b} x_{2}^{m}}$, when $b>m \geq 2$, is

$$
\begin{aligned}
\widehat{\left\langle x_{1}^{b} x_{2}^{m}\right\rangle}= & \sum_{i=1}^{m-1}(-1)^{b+m+1-i}(i) a^{3(b+m)-4 i}+(-1)^{b+1}(m-1) a^{3 b-m} \\
& +m \sum_{i=1}^{b-m-1}(-1)^{b+1-i} a^{3 b-m-4 i}+(-1)^{m+1}(m-1) a^{-b+3 m} \\
& +\sum_{i=1}^{m-2}(-1)^{m+1-i}(m-i) a^{-b+3 m-4 i}+2 a^{-b-m+4}+a^{-b-m-4} .
\end{aligned}
$$

Proof. We first verify the result for arbitrary $b$ and $m=2$ :

Resolving all $2^{3+2}$ crossings as were resolved for $\left\langle\widehat{x_{1}^{2} x_{2}^{2}}\right\rangle$ in Proposition 3.3, we get

$$
\left.\widehat{\left\langle x_{1}^{3} x_{2}^{2}\right.}\right\rangle=-a^{11}+a^{7}-a^{3}+2 a^{-1}+a^{-9}
$$


Similarly, we get

$$
\begin{aligned}
\left.\widehat{\left\langle x_{1}^{4} x_{2}^{2}\right.}\right\rangle & =a^{14}-a^{10}+2 a^{6}-a^{2}+2 a^{-2}+a^{-10} \\
& =-a^{3}\left\langle\widehat{x_{1}^{3} x_{2}^{2}}\right\rangle+a^{6}+a^{2}+2 a^{-2}+a^{-10}+a^{-6} \\
\left.\widehat{\left\langle x_{1}^{5} x_{2}^{2}\right.}\right\rangle & =-a^{17}+a^{13}-2 a^{9}+2 a^{5}-a+2 a^{-3}+a^{-11} \\
& =-a^{3}\left\langle\widehat{x_{1}^{4} x_{2}^{2}}\right\rangle+a^{5}+a+2 a^{-3}+a^{-11}+a^{-7} \\
\left.\widehat{\left\langle x_{1}^{6} x_{2}^{2}\right.}\right\rangle & =a^{20}-a^{16}+2 a^{12}-2 a^{8}+2 a^{4}-1+2 a^{-4}+a^{-12} \\
& =-a^{3}\left\langle\widehat{x_{1}^{5} x_{2}^{2}}\right\rangle+a^{4}+1+2 a^{-4}+a^{-8}+a^{-12}
\end{aligned}
$$

It follows from (3.11), (3.12), and (3.13) that

$$
\left.\widehat{\left\langle x_{1}^{b} x_{2}^{2}\right.}\right\rangle=-a^{3}\left\langle\widehat{x_{1}^{b-1} x_{2}^{2}}\right\rangle+a^{-b+10}+a^{-b+6}+2 a^{-b+2}+a^{-b-2}+a^{-b-6} .
$$

Now suppose the result is true for $b=t$ and $m=2$, that is

$$
\begin{aligned}
\left.\widehat{\left\langle x_{1}^{t} x_{2}^{2}\right.}\right\rangle= & (-1)^{-t+2} a^{3 t+2}+(-1)^{t+1} a^{3 t-2}+2 \sum_{i=1}^{t-3}(-1)^{t+1-i} a^{3 t-2-4 i} \\
& -a^{-t+6}+2 a^{-t+2}+a^{-t-6} .
\end{aligned}
$$

For $b=t+1$, we have

$$
\begin{aligned}
\left\langle\widehat{\left.x_{1}^{t+1} x_{2}^{2}\right\rangle}=\right. & -a^{3}\left\langle\widehat{x_{1}^{t} x_{2}^{2}}\right\rangle+a^{-t+9}+a^{-t+5}+2 a^{-t+1}+a^{-t-3}+a^{-t-7} \\
= & -a^{3}\left[(-1)^{-t+2} a^{3 t+2}+(-1)^{t+1} a^{3 t-2}+2 \sum_{i=1}^{t-3}(-1)^{t+1-i} a^{3 t-2-4 i}\right. \\
& \left.-a^{-t+6}+2 a^{-t+2}+a^{-t-6}\right]+a^{-t+9}+a^{-t+5}+2 a^{-t+1} \\
& +a^{-t-3}+a^{-t-7} \\
= & (-1)^{t+3} a^{3 t+5}+(-1)^{t+2} a^{3 t+1}+2 \sum_{i=1}^{t-3}(-1)^{t+2-i} a^{3 t+1-4 i} \\
& +a^{-t+9}-2 a^{-t+5}-a^{-t-3}+a^{-t+9}+a^{-t+5}+2 a^{-t+1} \\
& +a^{-t-3}+a^{-t-7} \\
= & (-1)^{t+3} a^{3 t+5}+(-1)^{t+2} a^{3 t+1} \\
& +\left[2 \sum_{i=1}^{t-3}(-1)^{t+2-i} a^{3 t+1-4 i}+2 a^{-t+9}\right] \\
& -a^{-t+5}+2 a^{-t+1}+a^{-t-7} \\
= & (-1)^{(t+1)+2} a^{3(t+1)+2}+(-1)^{(t+1)+1} a^{3(t+1)-2} \\
& +2 \sum_{i=1}^{(t+1)-3}(-1)^{(t+1)+1-i} a^{3(t+1)-2-4 i} \\
& -a^{-(t+1)+6}+2 a^{-(t+1)+2}+a^{-(t+1)-6} .
\end{aligned}
$$


Similarly, we get

$$
\begin{aligned}
\widehat{\left\langle x_{1}^{b} x_{2}^{3}\right\rangle}= & \sum_{i=1}^{2}(-1)^{b+4-i}(i) a^{3 b+9-4 i}+(-1)^{b+1} 2 a^{3 b-3} \\
& +3 \sum_{i=1}^{b-4}(-1)^{b+1-i} a^{3 b-3-4 i}+2 a^{-b+9} \\
& -a^{-b+5}+2 a^{-b+1}+a^{-b-7}
\end{aligned}
$$

and

$$
\begin{aligned}
\left.\widehat{x_{1}^{b} x_{2}^{4}}\right\rangle= & \sum_{i=1}^{3}(-1)^{b+5-i}(i) a^{3 b+12-4 i}+(-1)^{b+1} 3 a^{3 b-4} \\
& +4 \sum_{i=1}^{b-5}(-1)^{b+1-i} a^{3 b-4-4 i}-3 a^{-b+12} \\
& +\sum_{i=1}^{2}(-1)^{5-i}(4-i) a^{-b+12-4 i}+2 a^{-b}+a^{-b-8} .
\end{aligned}
$$

Now with the assumption that the result is true for an arbitrary $m$, we have

$$
\begin{aligned}
& \left\langle\widehat{x_{1}^{b} x_{2}^{m+1}}\right\rangle \\
& =\quad-a^{3} \widehat{\left\langle x_{1}^{b} x_{2}^{m}\right\rangle}+(-1)^{b} a^{3 b-(m+1)+4}+\sum_{i=1}^{b-3}(-1)^{b+1-i}(i) a^{3 b-(m+1)-4 i} \\
& +2 a^{-b-(m+1)+4}+a^{-b-(m+1)}+a^{-b-(m+1)-4} \\
& =\quad \sum_{i=1}^{m-1}(-1)^{b+m+2-i}(i) a^{3(b+m)+3-4 i}+(-1)^{b+2}(m-1) a^{3 b-m+3} \\
& +m \sum_{i=1}^{b-m-1}(-1)^{b+2-i} a^{3 b-m+3-4 i}+(-1)^{m+2}(m-1) a^{-b+3 m+3} \\
& +\sum_{i=1}^{m-2}(-1)^{m+2-i}(m-i) a^{-b+3 m+3-4 i}-2 a^{-b-m+7}-a^{-b-m-1} \\
& +(-1)^{b} a^{3 b-(m+1)+4}+\sum_{i=1}^{b-3}(-1)^{b+1-i}(i) a^{3 b-(m+1)-4 i} \\
& +2 a^{-b-(m+1)+4}+a^{-b-(m+1)}+a^{-b-(m+1)-4} \\
& =\quad \sum_{i=1}^{(m+1)-1}(-1)^{b+(m+1)+1-i}(i) a^{3(b+(m+1))-4 i}
\end{aligned}
$$




$$
\begin{aligned}
& +m \sum_{i=1}^{b-m-1}(-1)^{b+2-i} a^{3 b-m+3-4 i}+(-1)^{m+2}(m-1) a^{-b+3 m+3} \\
& +\sum_{i=1}^{m-2}(-1)^{m+2-i}(m-i) a^{-b+3 m+3-4 i}-2 a^{-b-m+7} \\
& +\sum_{i=1}^{b-3}(-1)^{b+1-i}(i) a^{3 b-(m+1)-4 i}+2 a^{-b-(m+1)+4}+a^{-b-(m+1)-4} \\
& =\quad \sum_{i=1}^{(m+1)-1}(-1)^{b+(m+1)+1-i}(i) a^{3(b+(m+1))-4 i} \\
& +\left[(-1)^{b+1} m a^{3 b-m-1}+(-1)^{b} m a^{3 b-m-5}+(-1)^{b-1} m a^{3 b-m-9}\right. \\
& \left.+\cdots+(-1)^{m+3} m a^{-b+3 m+7}+\right]+(-1)^{m+2}(m-1) a^{-b+3 m+3} \\
& +\left[(-1)^{m+1}(m-1) a^{-b+3 m-1}+(-1)^{m}(m-1) a^{-b+3 m-5}\right. \\
& \left.+(-1)^{m-1}(m-3) a^{-b+3 m-9}+\cdots+(-1)^{4} 2 a^{-b-m+11}+\right] \\
& -2 a^{-b-m+7} \\
& +\left[\left((-1)^{b} a^{3 b-m-5}+(-1)^{b-1} a^{3 b-m-9}+\cdots+(-1)^{m+3} a^{-b+3 m+7}\right)\right. \\
& +\left((-1)^{m+2} a^{-b+3 m+3}+(-1)^{m+1} a^{-b+3 m-1}+(-1)^{m} a^{-b+3 m-5}\right. \\
& \left.\left.+\cdots+(-1)^{4} a^{-b-m+11}\right)\right]+2 a^{-b-(m+1)+4}+a^{-b-(m+1)-4}
\end{aligned}
$$

Now collecting terms of same exponents, we get

$$
\begin{aligned}
= & \sum_{i=1}^{(m+1)-1}(-1)^{b+(m+1)+1-i}(i) a^{3(b+(m+1))-4 i}+(-1)^{b+1} m a^{3 b-m-1} \\
& +\left[+(-1)^{b}(m+1) a^{3 b-m-5}+(-1)^{b-1}(m+1) a^{3 b-m-9}\right. \\
& \left.+\cdots+(-1)^{m+3}(m+1) a^{-b+3 m+7}+\right]+(-1)^{m+2}(m) a^{-b+3 m+3} \\
& +\left[(-1)^{m+1}(m) a^{-b+3 m-1}+(-1)^{m}(m-1) a^{-b+3 m-5}\right. \\
& \left.+\cdots+(-1)^{4} 3 a^{-b-m+11}-2 a^{-b-m+7}\right] \\
& +2 a^{-b-(m+1)+4}+a^{-b-(m+1)-4}
\end{aligned}
$$

which finally, in terms of summation form, is the required result. 
Theorem 3.6. For any $b, m \geq 2$,

$$
\left.\widehat{\left\langle x_{1}^{b} x_{2}^{m}\right.}\right\rangle=\left\langle\widehat{x_{1}^{b}}\right\rangle\left\langle\widehat{x_{1}^{m}}\right\rangle .
$$

Proof. Depending on $b$ and $m$, the proof is divided into three cases: when $b, m$ are even and equal, when $b, m$ are odd and equal, and when $b, m$ are distinct.

Case I. (When $b$ and $m$ are even and equal.)

In this case, letting $m=b$, we show that $\left\langle\widehat{x_{1}^{b} x_{2}^{m}}\right\rangle=\left\langle\widehat{x_{1}^{b}}\right\rangle\left\langle\widehat{x_{1}^{m}}\right\rangle$. So, we proceed as follows:

$$
\left.\left.\widehat{\left\langle x_{1}^{2} x_{2}^{2}\right.}\right\rangle=a^{8}+2+a^{-8}=\left(-a^{4}-a^{-4}\right)\left(-a^{4}-a^{-4}\right)=\widehat{\left\langle x_{1}^{2}\right.}\right\rangle\left\langle\widehat{x_{1}^{2}}\right\rangle .
$$

Also, we have

$$
\begin{aligned}
\left.\widehat{x_{1}^{4} x_{2}^{4}}\right\rangle & =a^{20}-2 a^{16}+3 a^{12}-2 a^{8}+3 a^{4}-2+2 a^{-4}+a^{-12} \\
& =\left(-a^{10}+a^{6}-a^{2}-a^{-6}\right)\left(-a^{10}+a^{6}-a^{2}-a^{-6}\right) \\
& \left.=\left\langle\widehat{x_{1}^{4}}\right\rangle \widehat{x_{1}^{4}}\right\rangle
\end{aligned}
$$

and

$$
\begin{aligned}
\widehat{\left\langle x_{1}^{6} x_{2}^{6}\right\rangle=} & a^{32}-2 a^{28}+3 a^{24}-4 a^{20}+5 a^{16}-4 a^{12}+5 a^{8}-4 a^{4} \\
& +3-2 a^{-4}+2 a^{-8}+a^{-16} \\
= & \left(-a^{16}+a^{12}-a^{8}+a^{4}-a^{0}-a^{-8}\right)\left(-a^{16}+a^{12}-a^{8}\right. \\
& \left.+a^{4}-a^{0}-a^{-8}\right)=\left\langle\widehat{x_{1}^{6}}\right\rangle\left\langle\widehat{x_{1}^{6}}\right\rangle .
\end{aligned}
$$

Now we assume that the result is true for $b=k$, that is

$$
\left\langle\widehat{x_{1}^{k} x_{2}^{k}}\right\rangle=\left\langle\widehat{x_{1}^{k}}\right\rangle\left\langle\widehat{x_{1}^{k}}\right\rangle \text {. }
$$

Since $\left\langle\widehat{x_{1}^{n}}\right\rangle=-a^{3(n)-2}+a^{3(n)-6}+a^{-2}\left(\left\langle\widehat{x_{1}^{n-2}}\right\rangle\right)$, we have

$$
\begin{aligned}
& \left.\left.\widehat{\left\langle x_{1}^{k+2}\right.}\right\rangle \widehat{x_{1}^{k+2}}\right\rangle=\left[-a^{3 k+4}+a^{3 k}+a^{-2}\left(\left\langle\widehat{x_{1}^{k}}\right\rangle\right)\right]\left[-a^{3 k+4}+a^{3 k}\right. \\
& \left.+a^{-2}\left(\left\langle\widehat{x_{1}^{k}}\right\rangle\right)\right] \\
& =a^{-4}\left[\left\langle\widehat{x_{1}^{k}}\right\rangle\right]^{2}+a^{6 k+8}-2 a^{6 k+4}+a^{6 k}-2 a^{3 k+2}\left\langle\widehat{x_{1}^{k}}\right\rangle \\
& +2 a^{3 k-2}\left\langle\widehat{x_{1}^{k}}\right\rangle \\
& =a^{-4}\left[\left\langle\widehat{\left.x_{1}^{k}\right\rangle}\right]^{2}+a^{6 k+8}-2 a^{6 k+4}+a^{6 k}+2 a^{6 k}-2 a^{6 k-4}\right. \\
& +2 a^{6 k-8}-2 a^{6 k-12}+\cdots-2 a^{2 k+12}+2 a^{2 k+8}+2 a^{2 k} \\
& -2 a^{6 k-4}+2 a^{6 k-8}-2 a^{6 k-12}+2 a^{6 k-16}-\cdots+2 a^{2 k+8} \\
& -2 a^{2 k+4}-2 a^{2 k-4} \\
& =a^{-4}\left[\left\langle\widehat{x}_{1}^{k}\right\rangle\right]^{2}+a^{6 k+8}-2 a^{6 k+4}+3 a^{6 k}-4 a^{6 k-4}+4 a^{6 k-8} \\
& -4 a^{6 k-12}+4 a^{6 k-16}-\cdots+4 a^{2 k+8}-2 a^{2 k+4}+2 a^{2 k} \\
& -2 a^{2 k-4} \text {. }
\end{aligned}
$$


Also

$$
\begin{aligned}
& \left.\left\langle x_{1}^{\widehat{k+2} x_{2}^{k+2}}\right\rangle=a^{-4}\left[\widehat{\left\langle x_{1}^{k} x_{2}^{k}\right.}\right\rangle\right]-\sum_{i=1}^{k-1} i(-1)^{i+1} a^{6 k-4 i-4} \\
& +\sum_{i=1}^{k-1} i(-1)^{i+1} a^{6 k-4 i+12}-2 a^{2 k-4} \\
& +\sum_{i=k}^{k+1} i(-1)^{i+1} a^{6 k-4 i+12}-k a^{2 k+4}+(k+1) a^{2 k} \\
& =a^{-4}\left[\left\langle\widehat{x_{1}^{k}}\right\rangle\right]^{2}-a^{6 k-8}+2 a^{6 k-12}-3 a^{6 k-16}+4 a^{6 k-20} \\
& -\cdots-(k-3)(-1)^{k-2} a^{2 k+8}-(k-2)(-1)^{k-1} a^{2 k+4} \\
& -(k-1)(-1)^{k} a^{2 k}+a^{6 k+8}-2 a^{6 k+4}+3 a^{6 k}-4 a^{6 k-4} \\
& +5 a^{6 k-8}-6 a^{6 k-12}+7 a^{6 k-16}-8 a^{6 k-20}+\cdots \\
& +(k-3)(-1)^{k-2} a^{2 k+24}+(k-2)(-1)^{k-1} a^{2 k+20} \\
& +(k-1)(-1)^{k} a^{2 k+16}-2 a^{2 k-4}+k(-1)^{k+1} a^{2 k+12} \\
& +(k+1)(-1)^{k+2} a^{2 k+8}-k a^{2 k+4}+(k+1) a^{2 k} \\
& =a^{-4}\left[\left\langle\widehat{x_{1}^{k}}\right\rangle\right]^{2}+a^{6 k+8}-2 a^{6 k+4}+3 a^{6 k}-4 a^{6 k-4}+4 a^{6 k-8} \\
& -4 a^{6 k-12}+4 a^{6 k-16}-\cdots+4 a^{2 k+8}-2 a^{2 k+4}+2 a^{2 k} \\
& -2 a^{2 k-4} \text {. }
\end{aligned}
$$

The result now follows from (3.15) and (3.16).

Case II. (When $b$ and $m$ are odd and equal.) Similar to Case I.

Case III. (When $b$ and $m$ are distinct.)

In order to prove this part let us agree on the terminology:

$$
\begin{aligned}
& \bar{x}_{n}=(-1)^{m+n} a^{3 m-(4 n-2)}, n=1,2, \ldots, m-1, \bar{x}_{m}=-a^{-m-2} \\
& \bar{y}_{l}=(-1)^{b+l} a^{3 b-(4 l-2)}, l=1,2, \ldots, b-1, \bar{y}_{b}=-a^{-b-2} \\
& i=1,2, \ldots, m, j=1,2, \ldots, b ; b \geq 2 \\
& \left.\widehat{\left\langle x_{1}^{b}\right.}\right\rangle\left\langle\widehat{x_{1}^{m}}\right\rangle \\
& =\sum_{i+j=2}^{m} \bar{x}_{i} \bar{y}_{j}+\sum_{i+j=m+1, i \neq m} \bar{x}_{i} \bar{y}_{j}+\left[\sum_{i+j=m+2, i \neq m} \bar{x}_{i} \bar{y}_{j}+\bar{x}_{m} \bar{y}_{1}\right. \\
& \left.+\sum_{i+j=m+3, i \neq m} \bar{x}_{i} \bar{y}_{j}+\bar{x}_{m} \bar{y}_{2}+\cdots+\sum_{i+j=b, i \neq m} \bar{x}_{i} \bar{y}_{j}+\bar{x}_{m} \bar{y}_{b-m-1}\right]
\end{aligned}
$$




$$
\begin{aligned}
& +\left[\sum_{i+j=b+1, i \neq 1, m} \bar{x}_{i} \bar{y}_{j}+\bar{x}_{m} \bar{y}_{b-m}\right]+\left[\left(\sum_{i+j=b+2, i \neq 2, m} \bar{x}_{i} \bar{y}_{j}+\bar{x}_{m} \bar{y}_{b-m+1}+\bar{x}_{1} \bar{y}_{b}\right)\right. \\
& +\left(\sum_{i+j=b+3, i \neq 3, m} \bar{x}_{i} \bar{y}_{j}+\bar{x}_{m} \bar{y}_{b-m+2}+\bar{x}_{2} \bar{y}_{b}\right)+\cdots \\
& +\left(\sum_{i+j=b+m-3, i \neq m-3, m} \bar{x}_{i} \bar{y}_{j}+\bar{x}_{m} \bar{y}_{b-4}+\bar{x}_{m-4} \bar{y}_{b}\right) \\
& \left.+\left(\bar{x}_{m-1} \bar{y}_{b-1}+\bar{x}_{m} \bar{y}_{b-3}+\bar{x}_{m-3} \bar{y}_{b}\right)+\left(\bar{x}_{m} \bar{y}_{b-2}+\bar{x}_{m-2} \bar{y}_{b}\right)\right] \\
& +\left(\bar{x}_{m} \bar{y}_{b-1}+\bar{x}_{m-1} \bar{y}_{b}\right)+\bar{x}_{m} \bar{y}_{b}
\end{aligned}
$$

Since this agrees with the result of Proposition 3.5, the proof is finished.

\section{Competing Interests}

The author(s) do not have any competing interests in the manuscript.

\section{REFERENCES}

1. Kauffman, L. H. (1987). State models and the Jones polynomial. Topology, 26(3), 395-407.

2. Nizami, A. R., Munir, M., Sohail, T., \& Usman, A. (2016). On the Khovanov Homology of 2-and 3-Strand Braid Links. Advances in Pure Mathematics, 6(06), 481-491.

3. Nizami, A. R., Munir, M., Saleem, U., \& Ramzan, A. (2014). A Recursive Approach to the Kauffman Bracket. Applied Mathematics, 5(17), 2746-2755.

4. Reidemeister, K. (1948). Knot theory, Chelsea Publ. Co., New York.

5. Artin, E. (1925, December). Theorie der zöpfe. In Abhandlungen aus dem Mathematischen Seminar der Universitt Hamburg (Vol. 4, No. 1, pp. 47-72). Springer Berlin/Heidelberg.

6. Artin, E. (1947). Theory of braids. Annals of Mathematics, 101-126.

7. Birman, J. S. (2016). Braids, Links, and Mapping Class Groups.(AM-82) (Vol. 82). Princeton University Press.

8. Murasugi, K. (2007). Knot theory and its applications. Springer Science \& Business Media.

9. Alexander, J. W. (1923). A lemma on systems of knotted curves. Proceedings of the National Academy of Sciences, 9(3), 93-95.

10. Kauffman, L. H. (1990). An invariant of regular isotopy. Transactions of the American Mathematical Society, 318(2), 417-471.

\section{Abdul Rauf Nizami}

Abdus Salam School of Mathematical Sciences, Government College University, Pakistan. e-mail: arnizami@sms.edu.pk 